\section{In vivo gene delivery in the retina using polyethylenimine}

\author{
Hsi-Wen Liao and King-Wai Yau \\ Johns Hopkins University School of Medicine, Baltimore, MD, USA
}

BioTechniques 42:285-288 (March 2007)

doi 10.2144/000112404

Virus-based methods are widely used in the mammalian nervous system for expressing genes (1) and for producing short hairpin RNAs (shRNAs) (2) to knock down genes by RNA interference (RNAi). In the latter case, only a percentage of the small interfering RNAs (siRNAs) can effectively silence their cognate target genes (3). Thus, multiple selections of siRNA are often required. This multiplicity can make the virus-based method time-, labor-, or cost-intensive, especially when compounded with the goal of targeting multiple genes. Also, not all neuronal types are susceptible targets of a viral carrier. For example, retinal ganglion cells (RGCs) are known so far to be readily transduced only by adenoassociated virus and lentiviruses (4-6). Here, we report the successful use of a polymer as a carrier to deliver shRNAexpressing plasmid DNA to these cells in vivo.

Nonviral carriers such as polymers are simple to use and can be safer than viral carriers. The cationic polymer polyethylenimine (PEI) has been used for transfection in vitro and in vivo mostly of non-neuronal tissues (7). We tested the efficiency of PEI/DNA polyplexes for transfecting RGCs in vivo, so chosen because these cells are adjacent to the vitreous and therefore are likely accessible to the polyplexes delivered by intravitreal injection.

A commercial vector (RNAi-Ready pSIREN-DNR-DsRed-Express; Clontech, Mountain View, CA, USA) expressing shRNA (driven by a human U6 promoter) and a reporter Discosoma red fluorescent protein (DsRed) [driven by a cytomegalovirus (CMV) promoter] was mixed with PEI (in vivo-jetPEITM; Polyplus Transfection, Illkirch, France) according to the manufacturer's instructions. In this study, the N:P ratio (i.e., the number of nitrogen residues of in vivo-jetPEI per DNA phosphate) used was 10 (e.g., $1 \mu \mathrm{g}$ DNA was mixed with $0.2 \mu \mathrm{L}$ in vivo-jetPEI). The PEI/DNA polyplex solution (1.2 $\mu \mathrm{L}$ for each eye) was carefully administered intravitreally (Figure 1A) from the posterior-temporal side of the eye of an anesthetized mouse via a no. 33 custom needle (1-inch-long/sharp point/type no. 2; Hamilton, Reno, NV, USA) on a $2.5-\mu \mathrm{L}$ Hamilton syringe. The resulting expression of DsRed was evident in many cells in the ganglion cell layer (Figure 1B). In the retinal cross-section, DsRed expression was confirmed to be in the ganglion cell layer, indicating that the intravitreally injected polyplexes were able to cross the optic nerve fiber layer (containing the axons of the ganglion cells) from the vitreous (Figure 1C).

We next examined the shRNA expression. We designed the shRNA to target melanopsin (8), the photopigment mediating the light response of the intrinsically photosensitive RGCs (ipRGCs) $(9,10)$. The intrinsic photosensitivity of the ipRGCs is required in order for the pupillary light reflex to reach completion at high irradiances, with melanopsin-knockout mice showing an incomplete pupil restriction in bright light (9). We hypothesized that knocking down melanopsin in the wildtype mouse retina should produce a similar phenotype. We chose an albino background (Balb/c) because the pupil size of melanopsin-knockout mouse with this background (B6.129.Balb/c) at high irradiances was significantly larger than that with a pigmented background (B6.129; unpublished observation). The underlying mechanism for this difference is unclear, but may reflect multiple defects associated with the albino locus, including abnormal axonal projections from the eye to the brain, an underdeveloped central retina, and a deficit of the rod system

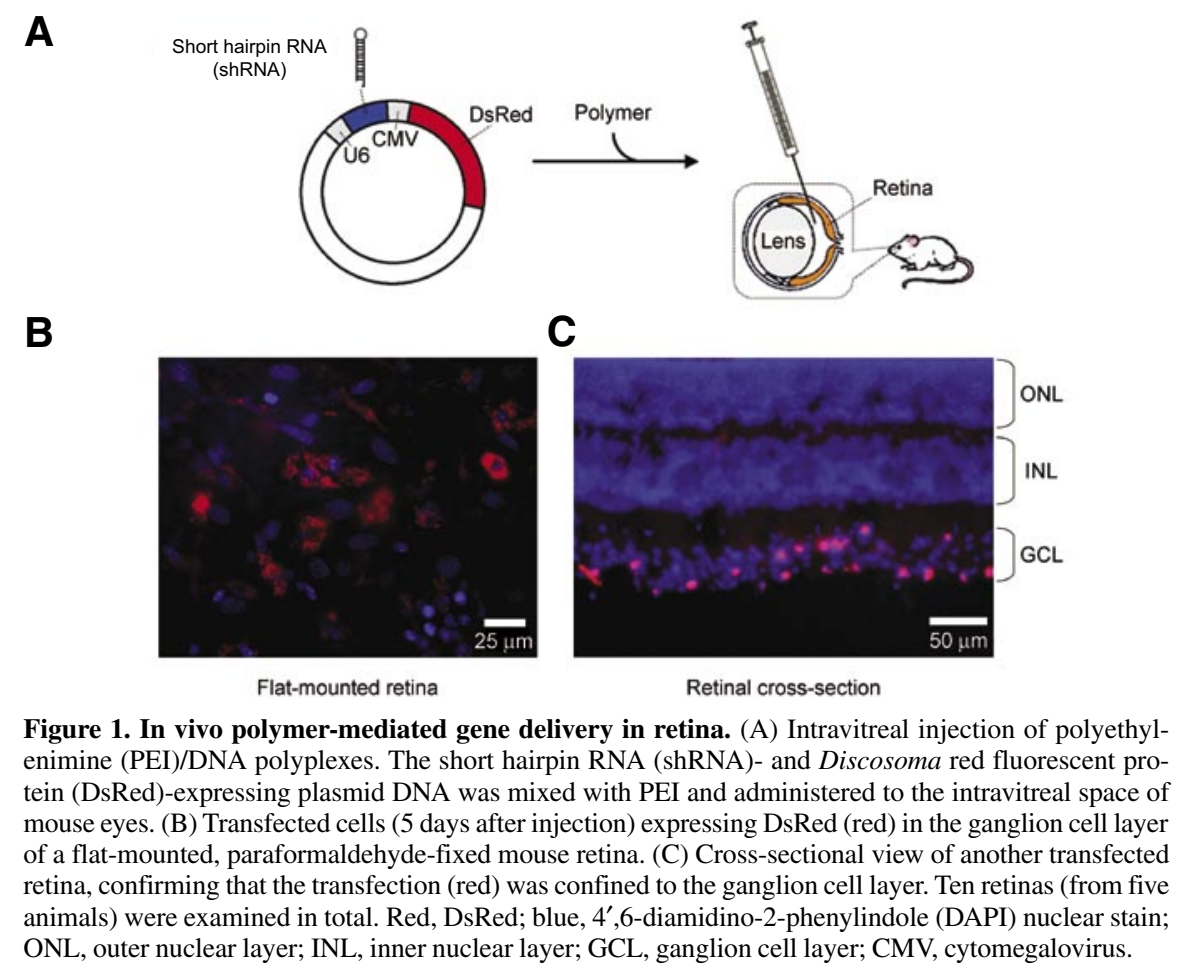




\section{Benchmarks}

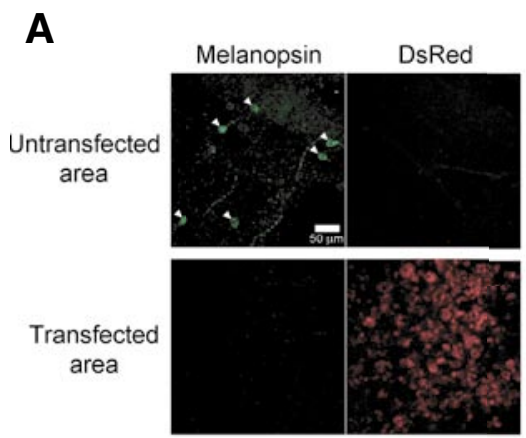

B

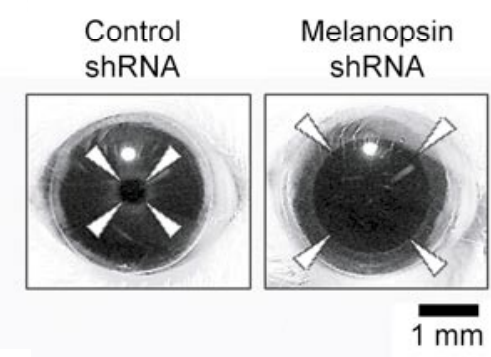

C

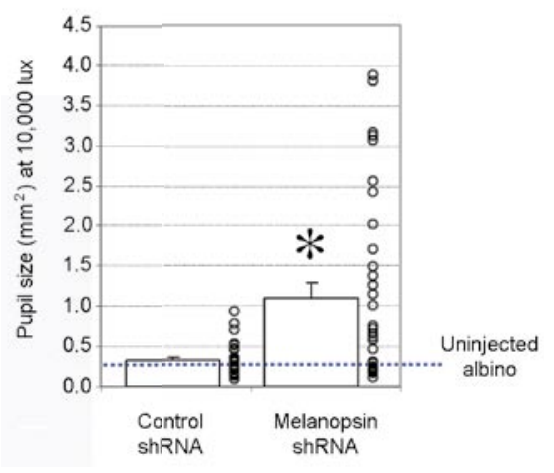

D

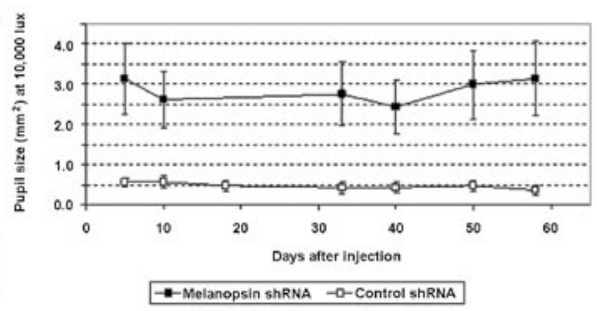

Figure 2. Melanopsin short hairpin RNA (shRNA) abolished melanopsin expression in transfected area of retina and decreased pupillary light reflex at high irradiances. (A) Confocal images of a flat-mounted mouse retina immunostained for melanopsin at 5 days after injection of melanopsin shRNA-expressing plasmid DNA. The expression (green) of the melanopsin protein was normal in intrinsically photosensitive retinal ganglion cells (ipRGCs; arrow heads) in the untransfected area, but absent in the transfected area (red). Ten retinas (from five animals) were examined. The red is Discosoma red fluorescent protein (DsRed). The green used was Alexa Fluor ${ }^{\circledR} 488$-conjugated secondary antibody (Molecular Probes; Invitrogen, Carlsbad, CA, USA). The sequences used for melanopsin shRNA were: 5'-ACGGTCATCTACACCTTCT-3', 5'-ATCATCAACCTCGCAGTCA-3', 5'-CCATTATCTACGCCATCAC-3' , and 5'-CCTAGAAGATGGAGAACTC-3'. All four were effective in knocking down melanopsin expression. The results shown in this panel were obtained with the first sequence. (B) Examples of pupil constriction at high irradiances (10,000 lux) of albino eyes injected with either control (left) or melanopsin (right) shRNA-expressing DNA construct at 5 days after injection. Arrow heads mark the boundary of pupil. The eye injected with melanopsin shRNA-expressing DNA construct showed a much more dilated pupil in bright light. The sequences used for control shRNA were for rod transducin: 5'-CATCATCTACGGCAACACT-3' and 5'-CGCTCAACATTCAGTATGG-3'; for cone transducin: 5'-AGGAAGCCAAGACTGTCAA- $3^{\prime}$ and 5'-GGAAGAGCACTATCGTCAA- ${ }^{\prime}$. Only one construct was injected to a given eye, and the collected results in panel $\mathrm{C}$ were pooled from all constructs. Both eyes of each mouse were injected with polyethylenimine (PEI)/DNA polyplexes. The measurements of pupil constriction were carried out 5 days after injection to allow enough time for the wounds caused by the injection to heal and the RNA interference (RNAi) to take effect. For some mice, measurements were also made at different time points to study the time course of the RNAi effect. Before the measurement of pupil constriction, the mice were kept in a 14/10 light/dark cycle. In room light (approximately 250-350 lux), a mouse (without anesthesia) was held by hand behind an opaque screen with a hole (slightly larger than the eye) at the center. The eye to be measured was placed against the hole to receive a steady intense light stimulus (10,000 lux; tungsten incandescent bulb, unfiltered) from the other side of the screen; the other eye (not stimulated by the bright light) remained exposed to room light. A photograph of the stimulated eye was taken 5-8 s after stimulus onset (when the pupil constriction had stabilized). Next, the other eye was stimulated and photographed similarly. The pupil size on the photograph was later measured manually using Adobe ${ }^{\circledR}$ Photoshop ${ }^{\circledR}$. The pupil reflex is bilaterally controlled $(12,16,17)$, so in principle, the eye unstimulated by the intense light but still exposed to the room light would contribute to the pupil constriction. However, the light intensity required for full pupil constriction far exceeded that of the room light. (C) Collective data of pupillary light reflex at high irradiances. Thirtysix eyes (18 animals) were used in each of the control shRNA and the melanopsin shRNA groups. The circles indicate measurements from individual eyes; bars indicate averages \pm standard errors. The asterisk indicates that the $P$ value of the Student's $t$-test $<0.001$. The blue dotted line marks the average pupil size of uninjected albino mice in the same light condition. (D) Long-lasting effect of melanopsin shRNA. The measurements were stopped at 2 months, while the knockdown effect still showed no sign of decrease. Six eyes (three animals) were used in each of the control shRNA (first sequence for rod transducin described above) and the melanopsin shRNA (first sequence for melanopsin) groups. Error bar, mean \pm SEM.

(11). The pupil reflex in an albino background thus gave a more dramatic indication of melanopsin knockdown. In Figure 2A, immunohistochemistry with a melanopsin antibody (12) indicated that melanopsin expression in the transfected (DsRed positive) area was reduced to an undetectable level by the melanopsin-specific shRNA. In the untransfected (DsRed negative) area of the same retina, melanopsin expression was normal. The eyes injected with melanopsin-shRNA-expressing plasmid DNA showed an incomplete pupil constriction in bright light $(10,000$ lux $)$, whereas the eyes injected with control DNA were unaffected (Figure 2, B and C). The variation in pupil constriction from eye to eye due to melanopsin-specific shRNA (Figure 2C) conceivably resulted from the variation in the size of the transfected area. Over the entire retina, the average number of remaining melanopsinimmunoreactive cells (all found outside the transfected area) at 5 days after transfection was $176 \pm 28$ (mean \pm SEM; 12 retinas from 6 animals), which translated to $25 \% \pm 4 \%$ of the total melanopsin-espressing RGCs (MOP-RGCs) (12). Thus, the average transfected area was $75 \% \pm 4 \%$ (mean \pm SEM). The variation in the transfected area presumably reflects the technically challenging intravitreal injection into the very limited space between the retina and the lens in mouse. In other mammals, including primates, the intravitreal injection should be considerably easier. Part of the variation in pupil size, which has autonomic input from the nervous system (9), could also have come from the animal's stress level during handling (13). Nonetheless, the melanopsin-knockdown effect was clear. The DsRed expression and the melanopsin-knockdown effect started to appear as early as $16 \mathrm{~h}$ after injection and lasted at least 2 months (Figure 2D).

We thus have demonstrated a fast and simple nonviral method using a polymer for delivering DNA to RGCs. From sequence design to injection, it can be as fast as a few days in the case of an RNAi experiment involving even multiple constructs. Recently, it has been shown possible to restore retinal photosensitivity in mice that 


\section{Benchmarks}

have degenerated rods and cones by the virus-mediated expression of channelrhodopsin, a photosensitive ion channel, in RGCs (14). Thus, there is considerable research interest in vision reviving gene therapy involving these cells. The current method provides a simple alternative approach. Conceivably, with injection into the subretinal space, the same method can be used for gene delivery to the rods and cones, another active area of research on ameliorating loss of vision associated with defects in rod/cone function (15). As mentioned earlier, the polymer method becomes particularly expedient when the deliveries of many DNA constructs have to be tested or made.

\section{ACKNOWLEDGMENTS}

We thank members of the Yau laboratory for helpful comments on the manuscript. This work was supported by grant no. EY14596 from the U.S. National Institutes of Health (NIH).

\section{COMPETING INTERESTS STATEMENT}

The authors declare no competing interests.

\section{REFERENCES}

1. Young, L.S., P.F. Searle, D. Onion, and V. Mautner. 2006. Viral gene therapy strategies: from basic science to clinical application. J. Pathol. 208:299-318.

2. Raoul, C., S.D. Barker, and P. Aebischer. 2006. Viral-based modelling and correction of neurodegenerative diseases by RNA interference. Gene Ther. 13:487-495.

3. Reynolds, A., D. Leake, Q. Boese, S. Scaringe, W.S. Marshall, and A. Khvorova. 2004. Rational siRNA design for RNA interference. Nat. Biotechnol. 22:326-330.

4. Dudus, L., V. Anand, G.M. Acland, S.J. Chen, J.M. Wilson, K.J. Fisher, A.M. Maguire, and J. Bennett. 1999. Persistent transgene product in retina, optic nerve and brain after intraocular injection of rAAV. Vision Res. 39:2545-2553.

5.Martin, K.R., R.L. Klein, and H.A. Quigley. 2002. Gene delivery to the eye using adeno-associated viral vectors. Methods 28:267-275.

6. van Adel, B.A., C. Kostic, N. Deglon, A.K. Ball, and Y. Arsenijevic. 2003. Delivery of ciliary neurotrophic factor via lentiviral- mediated transfer protects axotomized retinal ganglion cells for an extended period of time. Hum. Gene Ther. 14:103-115.

7. Lungwitz, U., M. Breunig, T. Blunk, and A. Gopferich. 2005. Polyethyleniminebased non-viral gene delivery systems. Eur. J. Pharm. Biopharm. 60:247-266.

8. Provencio, I., G. Jiang, W.J. De Grip, W.P. Hayes, and M.D. Rollag. 1998. Melanopsin: an opsin in melanophores, brain, and eye. Proc. Natl. Acad. Sci. USA 95:340-345.

9. Lucas, R.J., S. Hattar, M. Takao, D.M. Berson, R.G. Foster, and K.-W. Yau. 2003. Diminished pupillary light reflex at high irradiances in melanopsin-knockout mice. Science 299:245-247.

10.Fu, Y., H.-W. Liao, M.T. Do, and K.-W. Yau. 2005. Non-image-forming ocular photoreception in vertebrates. Curr. Opin. Neurobiol. 15:415-422.

11. Jeffery, G. 1998. The retinal pigment epithelium as a developmental regulator of the neural retina. Eye 12:499-503.

12. Hattar, S., H.-W. Liao, M. Takao, D.M. Berson, and K.-W. Yau. 2002. Melanopsincontaining retinal ganglion cells: architecture, projections, and intrinsic photosensitivity. Science 295:1065-1070.

13.Plouffe, L. and R.M. Stelmack. 1979. Neuroticism and the effect of stress on the pupillary light reflex. Percept. Mot. Skills 49:635-642.

14. Bi, A., J. Cui, Y.P. Ma, E. Olshevskava, M. Pu, A.M. Dizhoor, and Z.H. Pan. 2006. Ectopic expression of a microbial-type rhodopsin restores visual responses in mice with photoreceptor degeneration. Neuron 50:2333.

15. Dinculescu, A., L. Glushakova, S.H. Min, and W.W. Hauswirth. 2005. Adeno-associated virus-vectored gene therapy for retinal disease. Hum. Gene Ther. 16:649-663.

16. Trejo, L.J. and C.M. Cicerone. 1984. Cells in the pretectal olivary nucleus are in the pathway for the direct light reflex of the pupil in the rat. Brain Res. 300:49-62.

17. Clarke, R.J. and H. Ikeda. 1985. Luminance and darkness detectors in the olivary and posterior pretectal nuclei and their relationship to the pupillary light reflex in the rat. I. Studies with steady luminance levels. Exp. Brain Res. 57:224-232.

Received 9 October 2006; accepted 23 December 2006.

Address correspondence to Dr. Hsi-Wen Liao, Room 905, Preclinical Teaching Building, Johns Hopkins University School of Medicine, 725 N. Wolfe Street, Baltimore, MD 21205,USA.e-mail: hwliao@jhmi.edu

To purchase reprints of this article, contact: Reprints@BioTechniques.com 